\title{
Carbon Nanotubes Dispersion in Polymers by Two-Factor Mechanical Method
}

\author{
Sevda A. Abdullayeva',2, Asger B. Huseynov², Nahida N. Musayeva1,2, Rasim B. Jabbarov ${ }^{1,2}$, \\ Chingiz A. Sultanov², Akif D. Guliyev³, Rovshan F. Hasanov ${ }^{1,2}$
}

${ }^{1}$ G. M. Abdullayev Institute of Physics, Azerbaijan NAS, Baku, Azerbaijan

${ }^{2}$ Research \& Development Center for Hi-Technologies, MCIT, Baku, Azerbaijan

${ }^{3}$ Y. Mammadaliyev Institute of Petrochemical Processes, Azerbaijan NAS, Baku, Azerbaijan

Email: nmusayeva@physics.ab.az

How to cite this paper: Abdullayeva, S.A., Huseynov, A.B., Musayeva, N.N., Jabbarov, R.B., Sultanov, Ch.A., Guliyev, A.D. and Hasanov, R.F. (2016) Carbon Nanotubes Dispersion in Polymers by Two-Factor Mechanical Method. Advances in Materials Physics and Chemistry, 6, 291-304. http://dx.doi.org/10.4236/ampc.2016.611028

Received: October 19, 2016

Accepted: November 21, 2016

Published: November 24, 2016

Copyright $\odot 2016$ by authors and Scientific Research Publishing Inc. This work is licensed under the Creative Commons Attribution International License (CC BY 4.0).

http://creativecommons.org/licenses/by/4.0/

\begin{abstract}
The article reports the results on the development of highly optimized two-factor mechanical method of dispersion of multiwalled carbon nanotubes (MWCNTs) in polymer matrixes with the aim of preparing of nanocomposites. The investigation of the MWCNTs and nanocomposites was carried out by using scanning electron microscopy, X-ray diffraction and Raman spectroscopy. The effectiveness of the developed method is demonstrated by comparing the electrical conductivity of MWCNT/polymer nanocomposites prepared on the basis of polydimethylsiloxane (PDMS) and the epoxy resin, using two methods-the known chemical and the proposed one-two-factor mechanical.
\end{abstract}

\section{Keywords}

MWCNTs, Aerosol-CVD, Polymer, PDMS, Epoxy, Two-Factor Mechanical

\section{Introduction}

One of the main directions of development of the nanotechnology industry is the creation of new polymer composite materials with radically new properties through added components with nanoscale dimensions.

In recent years, as the most promising modifiers to create the electrically conductive nanocomposites were considered carbon nanotubes (CNTs), possessing a number of known unique physical and chemical properties [1] [2] [3]. The introduction and uniform distribution of the CNTs in a polymer matrix make it electrical conductive material, in addition increases their thermal conductivity, mechanical strength by several orders of magnitude [4] [5] [6]. 
During the manufacturing of high quality conductive polymer composites, main difficulties were bound with the necessity of a highly dispersed state of nanocarbon modifier and its uniform distribution in the polymer matrix.

Considering that nanotubes always tend to form agglomerates, the development of various processing methods for dispersing CNTs is required, which are different depending on the nature of the polymeric material and the type of carbon nanotubes themselves (functionalized or not, single or multiwalled, etc.). Functionalized CNTs with - $\mathrm{OH},-\mathrm{CHOH},-\mathrm{COOH},-\mathrm{NH}_{2}$, and other functional groups are often more consistent with the corresponding polymers forming the uniform composition of CNT/ polymer nanocomposites [7] [8] [9].

However, the use of the non functionalized CNTs in a polymer modifier is appropriate because of their lower cost in comparison with the functionalized and, moreover the formeris distinguished by the presence of effective modifying properties (for example high electrical conductivity), which are often drastically reduced in the functionalized process of CNTs [10].

In our study, we set a challenge-the development of effective two-factor mechanical method of obtaining the conductive polymer composites using not functionalized multiwalled CNTs as a modifier of the polymers and demonstration of their advantages compared to the known multi-step chemical method.

\section{Materials and Methods. Experiment}

Starting materials: as starting materials we used MWCNTs obtained by Aerosol CVD method and polymers-Polydimethylsiloxane (PDMS) and epoxy resins. Synthesis technology details of used MWCNTs have been demonstrated in [11] [12]. Cyclohexane and ferrocene in relative concentration of $20 \mathrm{mg} / \mathrm{ml}$ have been used as carbon source and catalyst, respectively. After synthesis of MWCNTs purification process with different solvents was done.

Purification of synthesized CNTs from tarry impurities was carried out using a solvent heated to $800 \mathrm{C}$-first with xylene and then with cyclohexane. Herewith the transparent xylene painted in yellowish color (Figure 1).

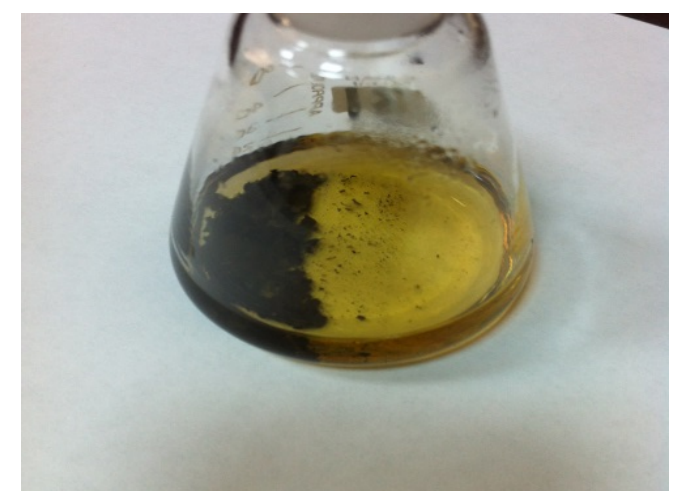

Figure 1. Purification process of synthesized MWCNTs in the xylene. 
X-ray diffraction patterns of purified MWCNTs are shown in Figure 2. The peak at $26.5^{\circ}$ corresponds to the interplanar spacing between the CNT walls and is marked as CNT (002) and second peak observed at 53.5 (004). The crystalline phase of -Fe is represented by a strong 44.5 (110) reflection, which is represented by an additional peak at $51.1^{\circ}$.

The intensity of the 002 peak associate with CNT is stronger than other $\mathrm{x}$-ray diffraction peaks due to presence of the more tangled nanotubes in the analysed sample (Figure 3(a)). The purification process does not lead to a reduction of length of separately nanotubes and deterioration of nanotube quality which is confirmed by both SEM and Raman spectroscopy analysis (Figure 3).

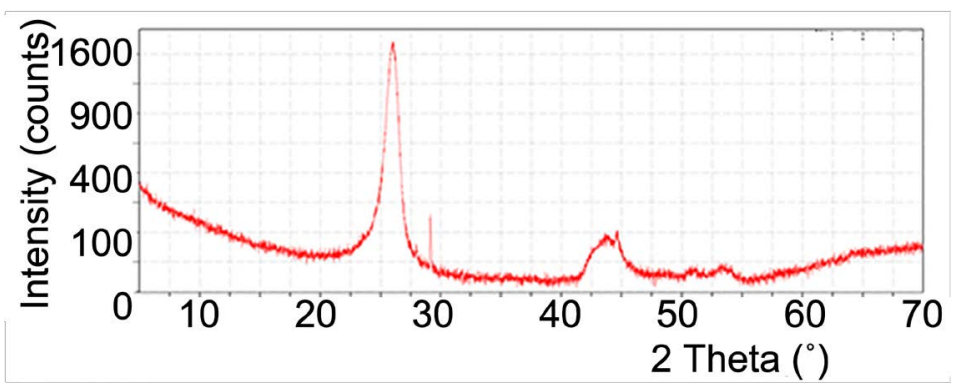

Figure 2. X-Ray Diffraction pattern of purified MWCNT sin xylene.

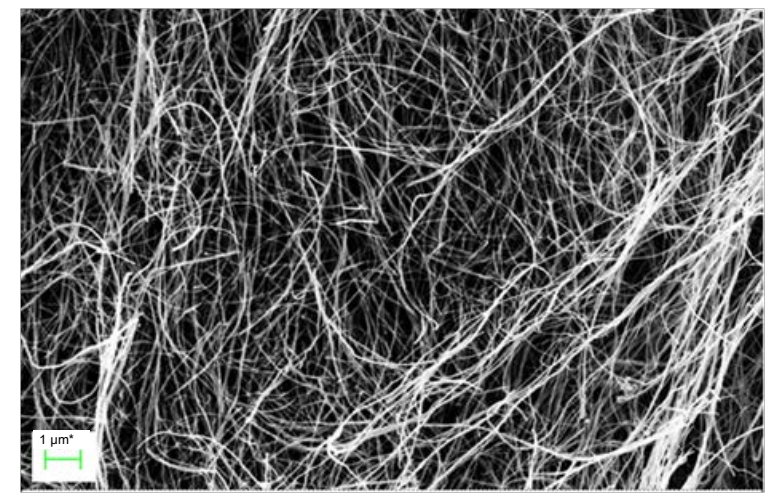

(a)

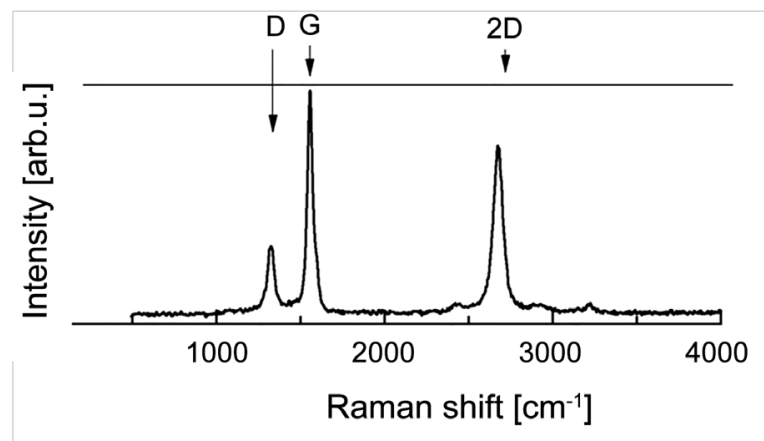

(b)

Figure 3. SEM picture (a) and Raman spectrum (b) of MWCNTs after purification. 


\subsection{Chemical Method of Dispersion}

There are different chemical and mechanical methods to prepare CNT/polymer nanocomposites [13] [14], depending on polymer properties, which is the main factor in the filling process. Anyway it is necessary to have high dispersion of CNTs in the polymer matrix and to create a network of CNTs, which play an important role in electrical conductivity of material [15].

In this chemical method is based on the chemical reaction between the oxidizing agents and CNT (functionalization process), further processing of the resultant product together with the polymer in a solvent by ultrasound then by distillation of solvent. Known mechanical method (melt mixing), which doesn't require the functionalization of CNTs based on mixing of MWCNTs in melts of polymers, but the technique requires an expensive equipment (mixer with melting) and has limitations as applicable only to thermoplastic polymers (polyethilene, polycarbonate, polyamide, and others), which melt without degradation, but the method cannot be applied to numerous types of polymers, relating to thermosetting.

To use chemical method [16] it is required to have functional groups on the CNTs (-OH, $-\mathrm{COH}, \mathrm{COOH}$ or $-\mathrm{NH}$ ). Purified MWCNTs were reacted with $\mathrm{H}_{2} \mathrm{SO}_{4}: \mathrm{HNO}_{3}$ (3:1) by using ultrasound mixing for 30 minutes and heating at $900 \mathrm{C}$ (to have much more functional groups this process is repeated 3 times), then product is washed by deionized water several times and filtered to $\mathrm{PH}=7$. Chloroform was used as solvent for dispersion after drying of the end product which is functionalized MWCNTs.

We assume that functionalization process leads to breaking of the nanotubes into several short ones under influence of acid, which can be observed on the SEM image (Figure 4).

The chloroform is evaporated from the solution during stirring the solution at $800 \mathrm{C}$ and polymerization process is then finished.

While the functionalized disperse well in PDMS by mentioned chemical method, there are several disadvantages of this method such as:

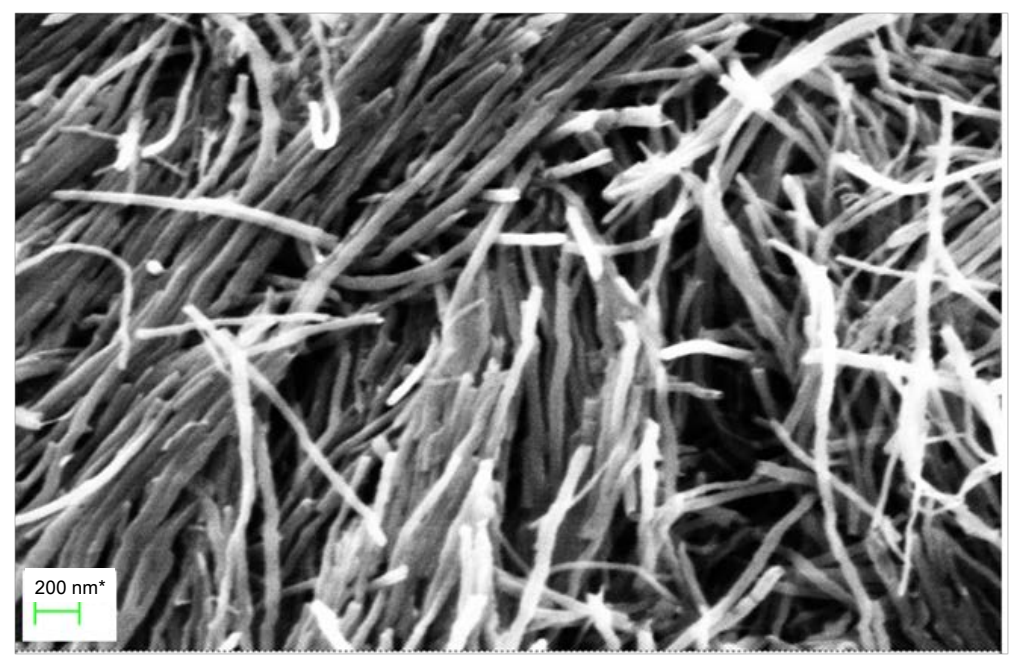

Figure 4. SEM picture of functionalized MWCNTs. 
1) The process is long and complicated.

2) Our experiments showed that the uniform dispersion of CNTs in PDMS is possible only at low percentage, and increasing of the relative percentage of CNTs in polymer has lead to its poor dispersion in the PDMS and creation of new agglomerates. In the literature there is practically no reliable information indicating a uniform dispersion of high percentage nonfunctionalized and also functionalized CNTs in the polymer by means of the mentioned chemical method.

3) This is very expensive, so it is not cost effective to implement large quantity samples.

\subsection{Two-Factor Mechanical Method of Dispersion}

For effective dispersion of non-functionalized CNTs was elaborated new two-factor mechanical method applicable to the solid polymers formed by polymerization of liquid monomers or oligomers (raw polymer material). Dispersion was carried out in the liquid phase, as a result of its subsequent polymerization-gave a solid composite: CNT/ polymer. The method is based on the simultaneous exposure of two factors:

1) Fine-mechanical destruction of existing agglomerates of nonfunctionalized CNTs to nano sized particles.

2) The presence of viscous liquid medium (monomers or oligomer with a suitable viscosity), preventing re-agglomeration of CNTs in the polymer matrix.

The first factor (the condition) is provided by two rubbing flat stainless steel surfaces with a small gap, no more than 200 microns, at a speed of one of the surfaces in the range of 400 - $500 \mathrm{rpm}$ (Figure 5).

The second factor (the necessary condition) is performed by selecting such monomers or oligomers that have optimum viscosity to prevent re-agglomeration of dispersed CNTs-a viscosity in the range of 0.5 - $10 \mathrm{~Pa} \cdot \mathrm{s}$. In this range of viscosities are many different widely used polymerizable resins such as epoxy, silicone, acrylic, etc.

Figure 5 shows a laboratory made dispersant for the preparation of polymer-carbon nanotube composite. The bottom plate with wide sides can be moved horizontally. The upper disc is connected to an electric motor with adjustable rotational speed and the

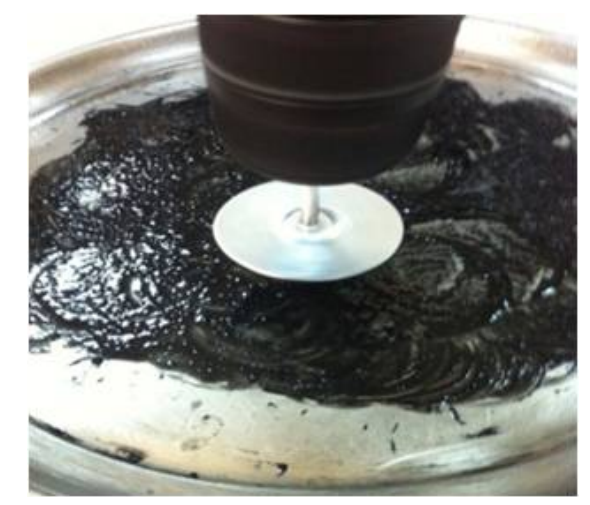

Figure 5. Dispersing process of nonfunctionalized CNTs in epoxy resin. 
vertical movement to establish the optimum gap between the plates. Sufficient duration of dispersion process (10 - 30 minutes) depends on the properties of the original polymer resin, and is selected empirically. Optimal dispersion temperatures are between $15^{\circ} \mathrm{C}-25^{\circ} \mathrm{C}$.

The polymerization of both Epoxy and PDMS require the hardener for each. Therefore was carried out 2 type experiments: First the hardener is mixed with polymers after fill it by MWCNTs. However, during the polymerization of already filled (with CNTs) polymer the hardener for it is required several times more than standard (10:1). The reason for this, probably, is the hindering effect of CNTs for a good contact of resin and hardener. And second hardener mixed with polymers before fill it by MWCNTs. Experiment results show that the second way is better for both dispersion of MWCNTs and polymerization (solidification) of the end composite. It is necessary to take into account the time of polymerization, which depends from the reaction temperature.

As mentioned above, the most important feature of this method is it doesn't need the functional groups on CNTs, which make it more interesting from scientific and practical point of view.

As a result, two types of nanocomposites CNT/PDMS and CNT/Epoxy with different percentage of CNTs have been prepared by both methods. The distribution of CNTs in polymer matrix has been analysed by Siqma HVSEM .

The samples of all prepared polymer nanocomposites have been cutted in shapes of rectangular parallelepipeds for the study of their electrical conductivity.

To create the connections directly on tops of our samples surface, in an easy, fast and reproducible way, the silver conductive paste was chosen [17], which does not require any particular equipment (such as metals sputtering or thermal evaporators devices) [18], just several minutes in order to let the solvent to evaporate. This way it was also prevented from any kind of warming of NCs or metals infiltrations during the metal contacts creation, which could have changed their properties. The electrical properties of nanocomposites have been studied by Hiresta-UPMCP-450 .

After obtaining the total current I under an applied voltage, the macroscopic electrical conductivity of the nanocomposite can be evaluated according to Ohm's law:

$\sigma_{\text {com }}=(\mathrm{I} / V) \times(D / S)$, where $D$ is the length between the two electrodes, and $S$ is the cross sectional area of the electrode.

\section{Results and Discussion}

The most accessible near-term application for CNTs-polymer composite involves their electrical properties. Two group polymers are available: dielectric and electrically conductive.

There are some polymer materials, which are electrically conductive due to some different mechanism [19]: 1) electron transport carried out by redox molecules that act as mobile carriers; 2) "hopping" electron transfer between redox groups linked to basic molecular framework or collected in supra-molecular associates at the expense of non-covalent interactions; 3) electronic conductivity along the system of conjugated 
five-bonds, which may include other groups capable of electron transfer, such as strained ring structures, hetero, having free, not involved in the formation of bonds electrons [20].

There are several ways to get conductive polymers:

The electronic structure of the polymer molecules with conjugated bonds in the unexcited state is in equilibrium, and their conductivity typically is very weak. In order to make such polymers electroconductive, they need electrochemically or chemically modified-“Doped”.

It is well known that the organic polymers obtained by the classical way with a high degree of coupling can be electroconductive. It is possible to achieve electroconductivity close to metal by means of inorganic doping [21].

The main disadvantages of these materials are the instability of their structure and the inability to control the distribution of the doping agent in the polymer. In addition, with these methods it is very difficult to obtain thin layers (coatings), which do not contain defects (holes), and this is sufficiently serious reason, which prevents from applying them in required areas.

Moreover, the necessity to use a doping substance in the preparation of the electrically conductive polymer [21] causes a difficulty, because these dopes are unstable when exposed to the environment, for example, iodine is able to evaporate from the coating, and others used doping compounds belong to the class of the Lewis acid and are easily hydrolyzed by air moisture, which determines the instability of the electrical conductivity of the material.

In this case, there is a need to develop more stable nanocomposites with high electrical conductivity.

The intrinsic high conductivity of CNTs makes them a logical choice for tuning the conductive properties of polymers. As demonstrated by MacDiarmid [22] CNTs are ideal candidate to fill the polymers because they can increase their electrical conductivity by many orders of magnitude from $10^{-10}-10^{-5}$ up to $10^{3}-10^{5} \mathrm{~S} / \mathrm{cm}$.

CNTs improve the strength properties of the polymer and make it a stable electroconductive material [14] [23].

However, there are some problems in preparation of nanocomposites. The biggest obstacle is the reformation of agglomerates of CNTs after their dispersing in the polymers. It set a new task to choose the right polymer and technology to disperse the CNTs in that polymer.

Using chemical method for dispersion of CNTs in PDMS it is observed that the agglomerates are negligible in the CNT/PDMS nanocomposites prepared by the chemical method in different percentage of CNTs ( $1 \%, 3 \%, 5 \%$ and $7 \%)$, that means dispersion is good (Figure 6).

Although the good dispersion is observed in the samples with $1 \%$ and $3 \%$ of CNTs in PDMS the electrical conductivity is very low $\left(<10^{-6} \mathrm{~S} / \mathrm{m}\right)$, even so as preventing from capturing of SEM pictures. Starting from 3\% CNTs electrical conductivity increases very rapidly (Figure 7). 
The samples obtained by the two-factor mechanical method demostrate good electrical properties even at $1 \%$ of CNTs in PDMS. SEM observation of the samples with same percentage (5\%) of CNTs in the PDMS shows that CNTs separated by mechanical influence are not fractured much and homogeneously distributed in the polymer (Figure 8), and the second (after dispersion) agglomeration process doesn't occur. This fact greatly influenced the electrical properties of the nanocomposite. As a fact we can show, that the electrical conductivity of the CNT/PDMS nanocomposite with $5 \%$ of CNTs, obtained by two-factor mechanical method, is $10^{3}-10^{4}$ times stronger than the nanocomposite with the same contents, obtained by chemical method (Figure 9).

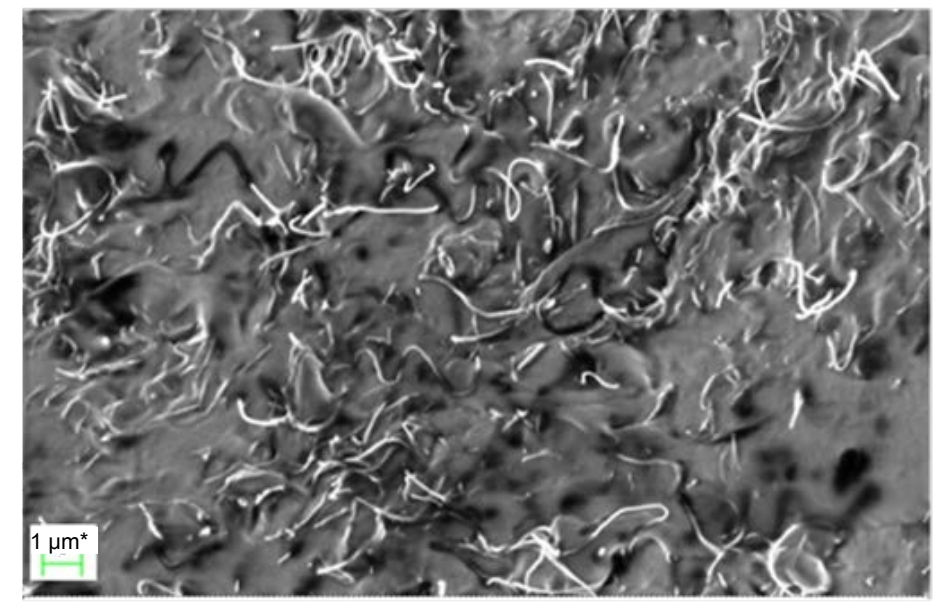

Figure 6. SEM picture of $5 \%$ MWCNT/PDMS nanocomposites obtained by chemical method.

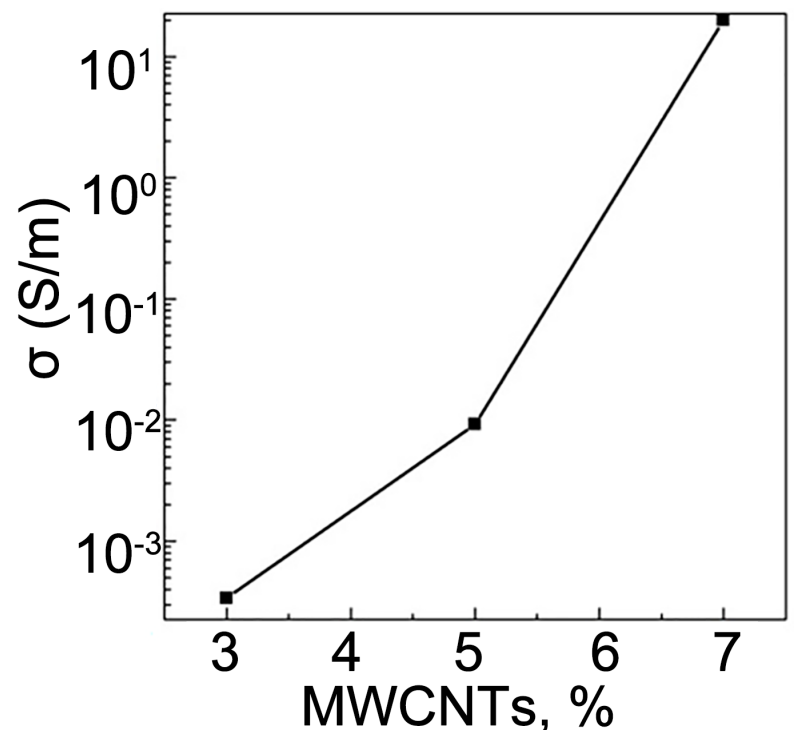

Figure 7. Electrical conductivity depending on the MWCNT content of MWCNT/PDMS nanocomposite obtained by the chemical method. 


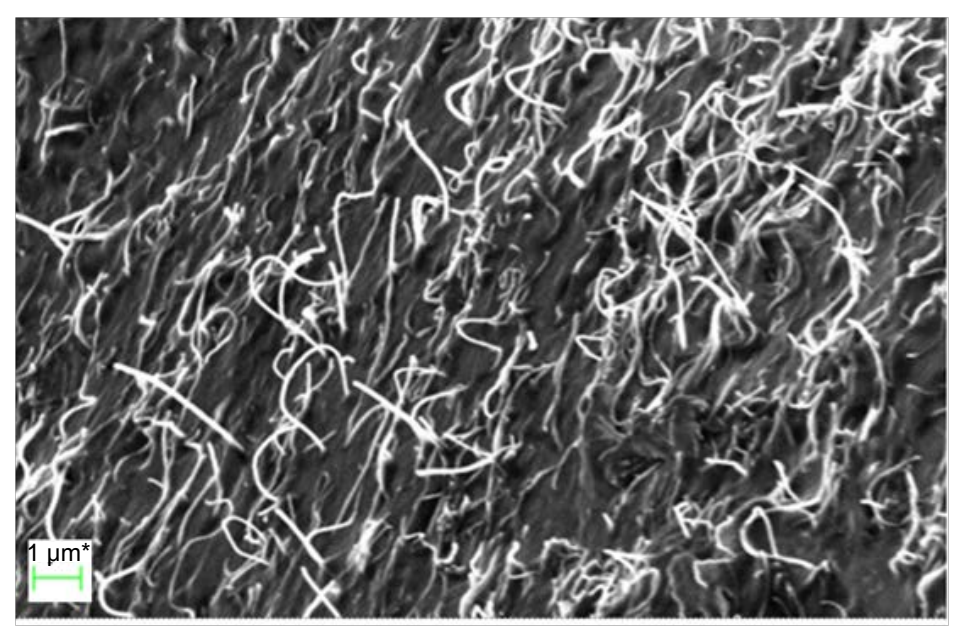

Figure 8. SEM picture of 5\% CNT/PDMS nanocomposites obtained by two-factor mechanical method.

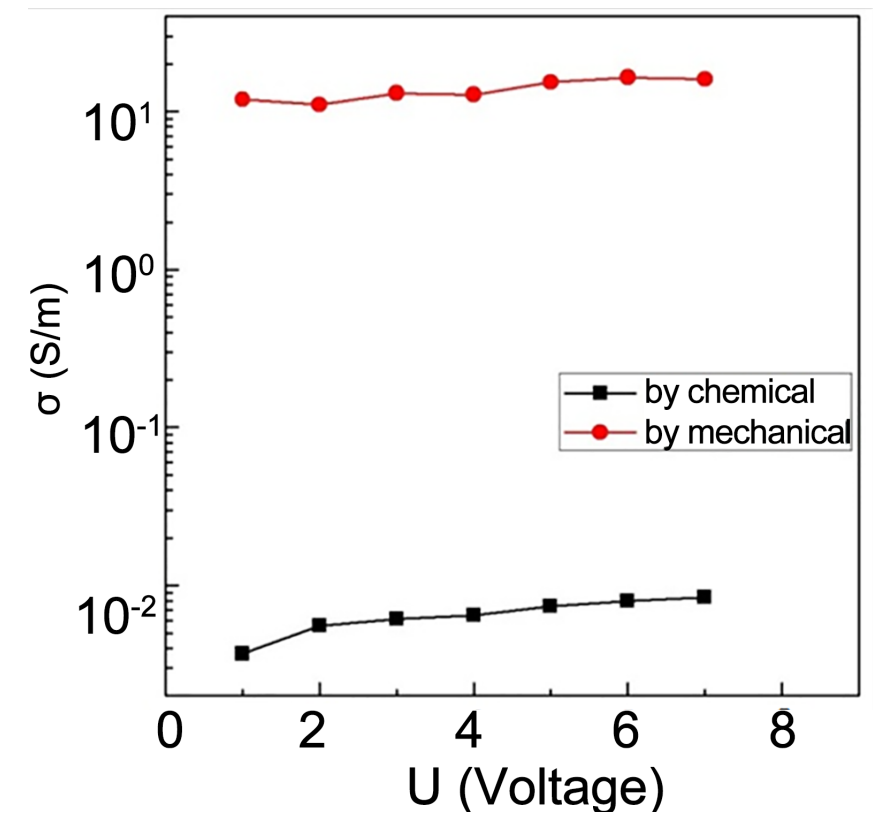

Figure 9. Comparison electric properties of the 5\% CNT/ PDMS nanocomposites obtained by chemical and two fac- tor mechanical methods.

CNT/Epoxy nanocomposites with the 1\%,2\%,3\% and 5\% percentage of MWCNTs have been prepared by both chemical and two-factor mechanical dispersion method and their electrical properties have been studied, too.

SEM analysis show that MWCNTs uniformly dispersed in Epoxy by two-factor mechanical method in contrast to the chemical method of dispersion (Figure 10)

As in the case of MWCNT/PDMS nanocomposite in the MWCNT/Epoxy, the electrical conductivity depending on the MWCNT content demonstrates sharp dependence of the current from filler concentration (Figure 11). 


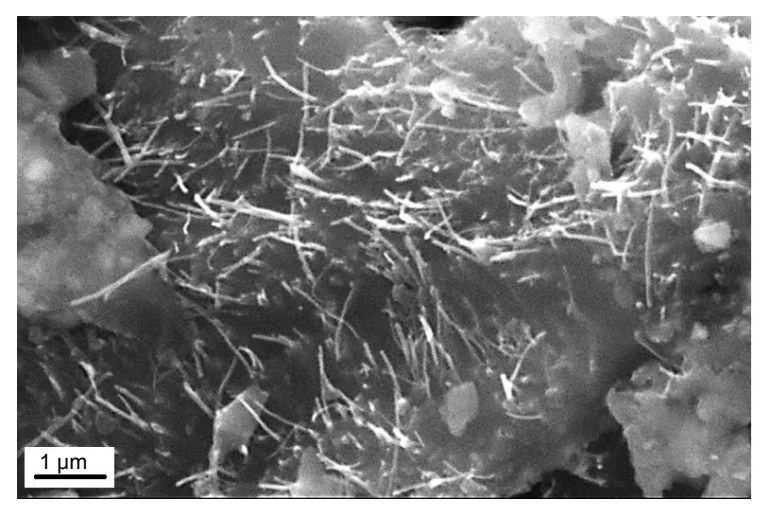

(a)

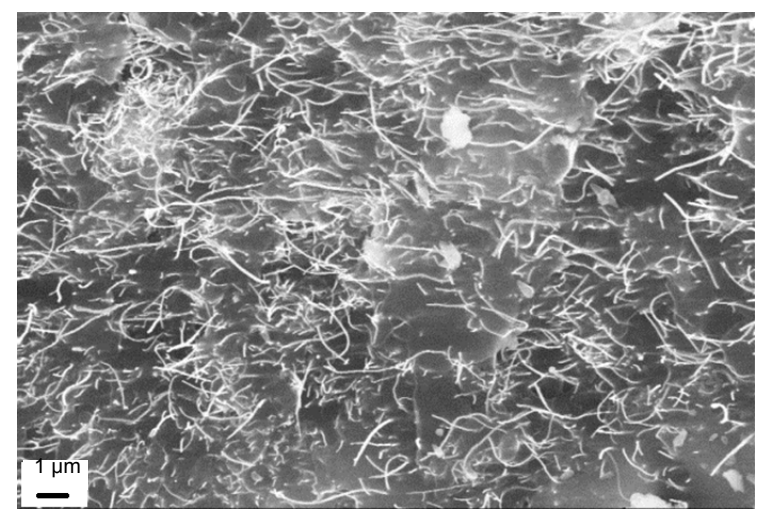

(b)

Figure 10. SEM pictures of the 5\% CNT/Epoxy nanocomposites obtained by chemical (a) and two- factor mechanical (b) methods.

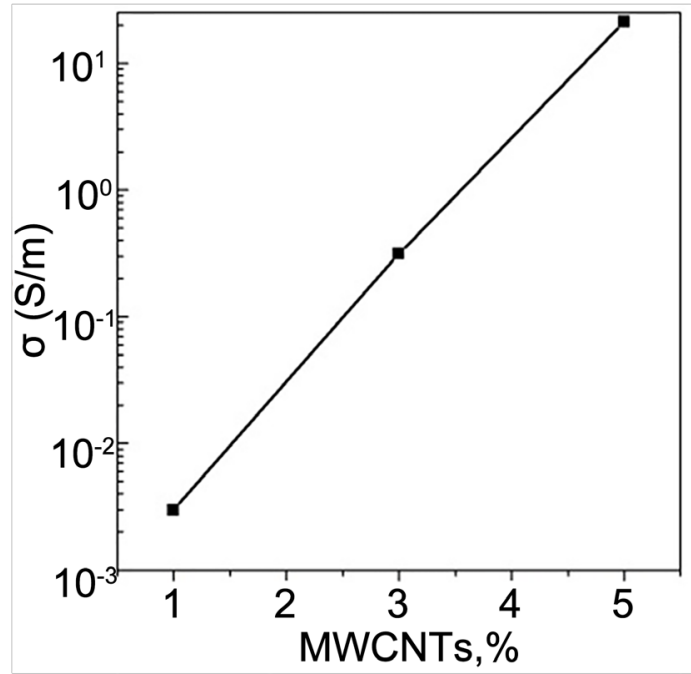

Figure 11. Electrical conductivity depending on the MWCNT content of the MWCNT/Epoxy nanocomposites obtained by two-factor mechanical method. 
The electrical conductivity of the MWCNT/Epoxy nanocomposite with $5 \%$ of CNTs, obtained by two-factor mechanical method, is $10^{4}-10^{5}$ times stronger than the nanocomposite with the same contents, obtained by chemical method (Figure 12).

The results were compared to the results of the authors G. Grimaldi et al. [24], who studied the electrical conductivity of composites made on the basis of MWCNTs and epoxy SU8. CNTs were dispersed in the SU8 by sonication in the presence of surfactant. PI was used for polymerization of the sample. The difference of their method from twofactor mechanical method is using of different surfactants to prepare the sample containing MWCNTs 5 mass $\%$. The electrical conductivity was about $10^{\circ} \mathrm{S} / \mathrm{m}$, which is 10 times less than the electrical conductivity of a 5\% of MWCNTs/Epoxy sample manufactured by two-factor mechanical method.

A similar comparison was made with the work of R. B. Mathur et al. [25], in which the authors used the functionalized multi-walled carbon nanotubes (MWCNT) as reinforcing material for thermoplastic polymer matrices, polymethyl methacrylate (PMMA), and polystyrene (PS). Dispersing procedure included the use of solvents (toluene, chloroform), and ultrasonic treatment. The authors were able to obtain high electrical conductivity of composites for both of the used polymers $\left(\sigma=10^{0} \mathrm{~S} / \mathrm{cm}\right)$ at $8-10 \mathrm{vol} . \%$ concentrations of MWCNTs. The mentioned electrical conductivity is slightly higher than the value achieved for the samples obtained by two-factor mechanical method ( $\sigma=$ $10^{1} \mathrm{~S} / \mathrm{m}$, for the concentrations of MWCNTs $=5 \%$ mas.). However, it should be noted that the composition of the polymers in the article was different from the polymers investigated by us, which caused difficulty in comparison of effective nesses of the dispersing methods, because the influence of the nature of polymer matrices took place. Moreover, non-functionalized CNT were used in the two-factor mechanical method developed by us, which excluded the laborious step of processing by concentrated acids.

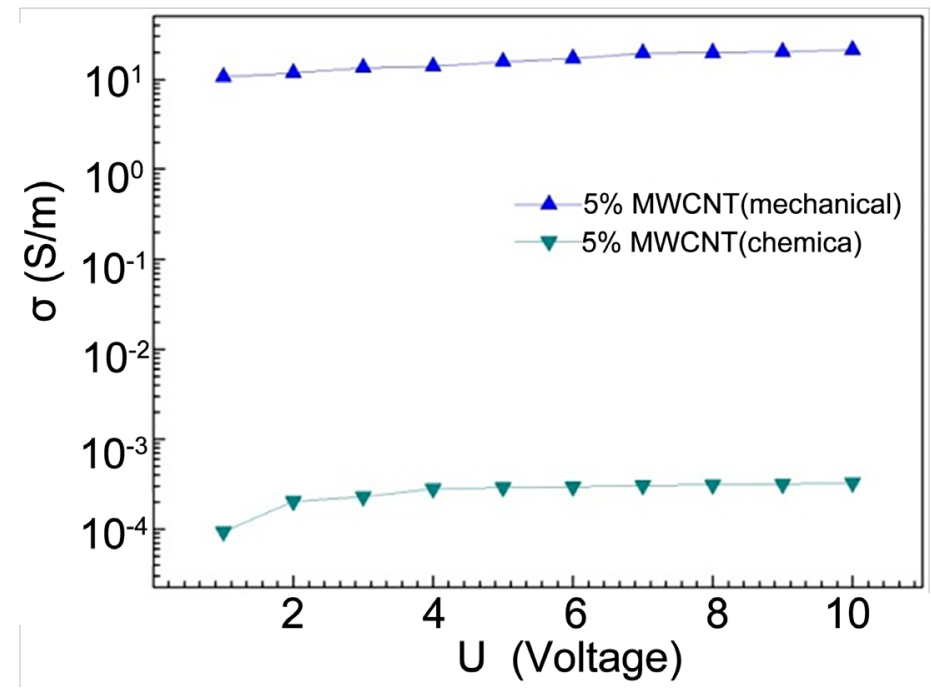

Figure 12. Comparison electric properties of the 5\% CNT/ Epoxy nanocomposites obtained by chemical and two-factor mechanical methods. 
Dispersion of high percentage CNTs was practically not possible by chemical method with the use of concentrated acids, while two-factor mechanical method allows to obtain nanocomposites with 20 mass\% CNTs dispersed in both PDMS and Epoxy. SEM pictures show uniform dispersion of 20 mass\% of CNTs in both polymers: PDMS and Epoxy (Figure 13).

\section{Conclusions}

In order to prepare nanocompositesa highly optimized, two-factor mechanical method for dispersion of multiwall carbon nanotubes (MWCNTs) in polymers has been developed, providing high uniformity of distribution of MWCNTs in a wide range of concentrations $(1 \mathrm{wt} \%-20 \mathrm{wt} \%)$ in the polymer matrix without degradation of the tubes during their dispersion.

It is revealed that in contrast to the two-factor mechanical method developed by us the chemical method is complex, more expensive and time-consuming, but also ineffective for the manufacture of composites with CNTs content greater than $10 \mathrm{wt} \%$.

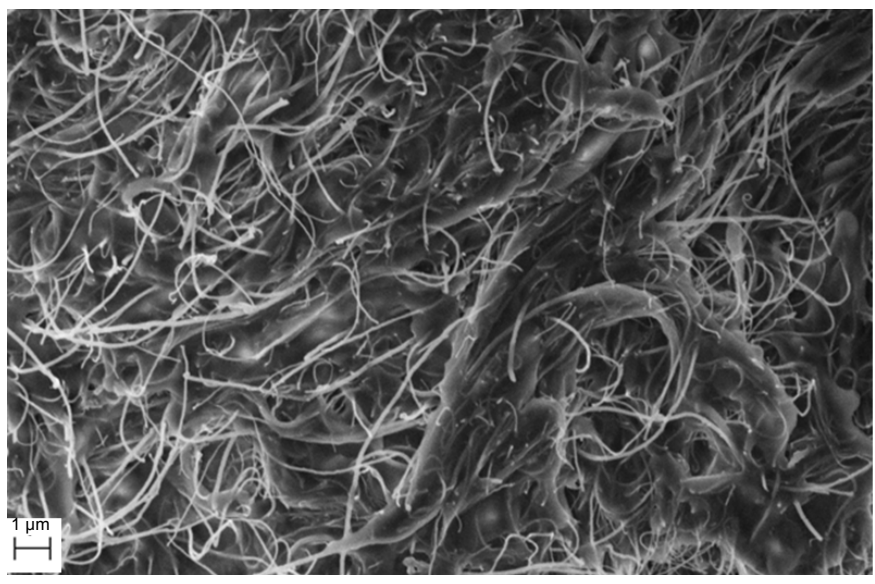

(a)

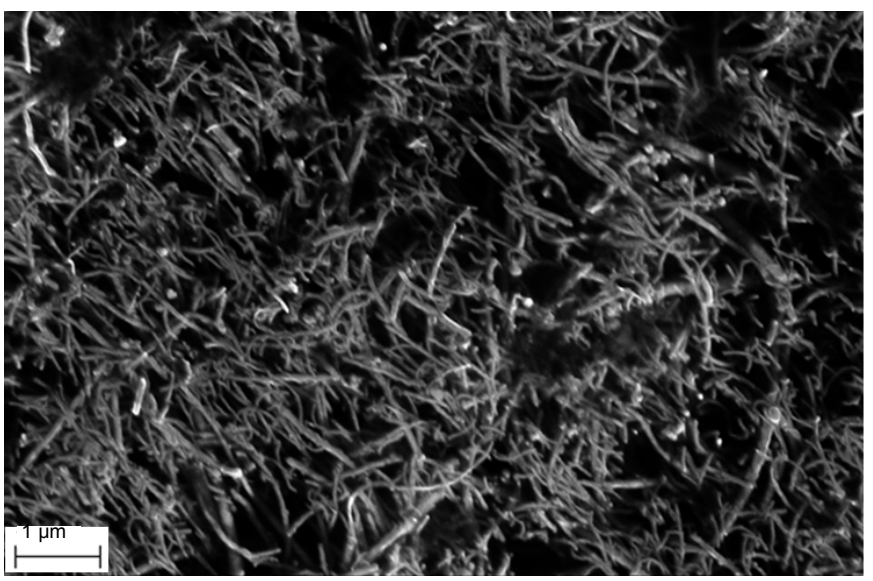

(b)

Figure 13. SEM pictures of 20 mass $\%$ of MWCNTs in both polymers PDMS (a) and Epoxy (b) by means of TFMM. 
SEM analysis shows that non functionalized MWCNTs disperse better by means of two-factor mechanical method, compared to the long-process chemical method, which was confirmed by electrical measurements. So, the electrical conductivity of the samples obtained by two factor mechanical method is $10^{3}-10^{5}$ times higher than the one's obtained by the chemical dispersion method.

\section{Acknowledgements}

Some authors of the paper are very grateful to the Scientific Foundation of the SOCAROil Company of Azerbaijan Republic, TWAS Organization and Bilateral project between IMEM-CNR and Institute of Physics-ANAS for partial financial support to carry out experiments.

\section{References}

[1] Suzuki, S. (2013) Physical and Chemical Properties of Carbon Nanotubes. InTech, 393.

[2] Charlier, J.C. and Roche, S. (2007) Electronic and Transport Properties of Nanotubes. Reviews of Modern Physics, 79, 677-732. https://doi.org/10.1103/RevModPhys.79.677

[3] Saito, R., Dresselhaus, G. and Dresselhaus, M.S. (1998) Physical Properties of Carbon Nanotubes. Imperial College Press, London, c1998, xii, 259 p. https://doi.org/10.1142/p080

[4] Yu, S., Juay, Y.K. and Young, M.S. (2008) Fabrication and Characterization of Carbon Nanotube Reinforced Poly(methyl methacrylate) Nanocomposites. Journal of Nanoscience and Nanotechnology, 8, 1852-1857. https://doi.org/10.1166/jnn.2008.011

[5] Miyagawa, H., Misra, M. and Mohanty, A.K. (2005) Mechanical Properties of Carbon Nanotubes and Their Polymer Nanocomposites. Journal of Nanoscience and Nanotechnology, 5, 1593-1615. https://doi.org/10.1166/jnn.2005.181

[6] Li, X., Gao, H., Scrivens, W.A., Fei, D., Xu, X., Sutton, M.A., Reynolds, A.P. and Myrick, M.L. (2007) Reinforcing Mechanisms of Single-Walled Carbon Nanotube-Reinforced Polymer Composites. Journal of Nanoscience and Nanotechnology, 7, 2309-2317. https://doi.org/10.1166/jnn.2007.410

[7] George, J.J., Sengupta, R. and Bhowmick, A.K. (2008) Influence of Functionalization of Multi-Walled Carbon Nanotubes on the Properties of Ethylene Vinyl Acetate Nanocomposites. Journal of Nanoscience and Nanotechnology, 8, 1913-1921.

https://doi.org/10.1166/jnn.2008.024

[8] Hou, Y., Tang, J., Zhang, H., Qian, C., Feng, Y.Y. and Liu, J. (2009) Functionalized FewWalled Carbon Nanotubes for Mechanical Reinforcement of Polymeric Composites. ACS Nano, 3, 1057-1062. https://doi.org/10.1021/nn9000512

[9] Pulikkathara, M.X., Kuznetsov, O.V., Peralta, I.R., Wei, X. and Khabashesku, V.N. (2009) Medium Density Polyethylene Composites with Functionalized Carbon Nanotubes. Nanotechnology, 20, 195602. https://doi.org/10.1088/0957-4484/20/19/195602

[10] Dyachkova, T.P., Melezhyk, A.V., Gorsky, S.Yu., Anosova, I.V. and Tkachev, A.G. (2013) Some Aspects of Functionalization and Modification of Carbon Nanomaterials. Nanosystems: Physics, Chemistry, Mathematics, 4, 605-621.

[11] Abdullayeva, S., Musayeva, N.N., Frigeri, C., Huseynov, A.B., Jabbarov, R.B., Abdullayev, R.B., Sultanov, Ch.A. and Hasanov, R.F. (2015) Characterization of High Quality Carbon Nanotubes Synthesized via Aerosol-CVD. Journal of Advances in Physics, 11, 3229-3240.

[12] Abdullayeva, S., Huseynov, A.B., Musayeva, N.N., Jabbarov, R.B., Sultanov, Ch.A. and 
Hasanov, R.F. (2016) Synthesis of Carbon Nanotubes Using Azerbaijan's Oil. Advances in Materials Physics and Chemistry, 6, 105-112. https:/doi.org/10.4236/ampc.2016.65011

[13] Alig, I., Pötschke, P., Lellinger, D., Skipa, T., Pegel, S., Kasaliwal, G.R. and Villmow, T. (2012) Establishment, Morphology and Properties of Carbon Nanotube Networks in Polymer Melts. Polymer, 53, 4-28. https:/doi.org/10.1016/j.polymer.2011.10.063

[14] Huyen, D.N. (2011) Chapter 22. Carbon Nanotubes and Semiconducting Polymer Nanocomposites. In: Yellampalli, S., Ed., Carbon Nanotubes-Synthesis, Characterization, Applications. Nanotechnology and Nanomaterials, InTech, 469-478. http://www.intechopen.com/

[15] Alig, I., Skipa, T., Lellinger, D. and Potschke, P. (2008) Destruction and Formation of a Carbon Nanotube Network in Polymer Melts: Rheology and Conductivity Spectroscopy. Polymer, 49, 3524-3532. https:/doi.org/10.1016/j.polymer.2008.05.037

[16] Liu, Ch.X. and Choi, J.W. (2012) Improved Dispersion of Carbon Nanotubes at High Concentrations. Nanomaterials, 2, 329-347. https://doi.org/10.3390/nano2040329

[17] Hu, N., Masuda, Z., Yan, Ch., Yamamoto, G., Fukunaga, H. and Hashida, T. (2008) The Electrical Properties of Polymer Nanocomposites with Carbon Nanotube Fillers. Nanotechnology, 19. https:/doi.org/10.1088/0957-4484/19/21/215701

[18] Coleman, J.N., Curran, S., Dalton, A.B., Davey, A.P., McCarthy, B., Blau, W. and Barklie, R.C. (1998) Percolation-Dominated Conductivity in a Conjugated-Polymer-Carbon- Nanotube Composite. Physical Review B, 58, 7492-7495. https:/doi.org/10.1103/PhysRevB.58.R7492

[19] Lupinski, J.H. and Kopple, K.D. (1964) Electroconductive Polymers. Science, 146, 10381039. https:/doi.org/10.1126/science.146.3647.1038

[20] Heeger, A.J. (2001) Semiconducting and Metallic Polymers: The Fousrth Generation of Polymeric Materials. The Journal of Physical Chemistry B, 105, 8475-8491. https:/doi.org/10.1021/jp011611w

[21] Gruenwald, H. and Munro, H.S. (1987) Process for the Preparation of Thin, Hole-Free, Electroconductive Polymer Layers. Patent DE3541721A1.

[22] MacDiarmid, A.G. (2001) "Synthetic Metals": A Novel Role for Organic Polymers (Nobel Lecture). Angewandte Chemie, 40, 2581-2590.

[23] Du, F.M., Scogna, R.C., Zhou, W., Brand, S., Fischer, J.E. and Winey, K.I. (2004) Nanotube Networks in Polymer Nanocomposites: Rheology and Electrical Conductivity. Macromolecules, 37, 9048-9055. https:/doi.org/10.1021/ma049164g

[24] Grimaldi, C., Mionić, M., Gaal, R., Forró, L. and Magrez, A. (2013) Electrical Conductivity of Multi-Walled Carbon Nanotubes-SU8 Epoxy Composites. Applied Physics Letters, 102, Article ID: 223114. https:/doi.org/10.1063/1.4809923

[25] Mathur, R.B., Pande, S., Singh, B.P. and Dhami, T.L. (2008) Electrical and Mechanical Properties of Multi-Walled Carbon Nanotubes Reinforced PMMA and PS Composites. Polymer Composites, 29, 717-727. 
Submit or recommend next manuscript to SCIRP and we will provide best service for you:

Accepting pre-submission inquiries through Email, Facebook, LinkedIn, Twitter, etc. A wide selection of journals (inclusive of 9 subjects, more than 200 journals)

Providing 24-hour high-quality service

User-friendly online submission system

Fair and swift peer-review system

Efficient typesetting and proofreading procedure

Display of the result of downloads and visits, as well as the number of cited articles

Maximum dissemination of your research work

Submit your manuscript at: http://papersubmission.scirp.org/

Or contact ampc@scirp.org 